\title{
Incidence, Prevalence, and Risk Factors of Hemiplegic Shoulder Pain: A Systematic Review
}

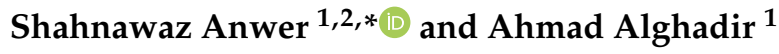 \\ 1 Department of Rehabilitation Sciences, College of Applied Medical Sciences, King Saud University, \\ Riyadh 11433, Saudi Arabia; aalghadir@hotmail.com \\ 2 Department of Building and Real Estate, Hong Kong Polytechnic University, Kowloon, Hong Kong \\ * Correspondence: anwerphysio@gmail.com
}

Received: 20 May 2020; Accepted: 30 June 2020; Published: 9 July 2020

\begin{abstract}
The current systematic review aimed to investigate the incidence, prevalence, and risk factors causing hemiplegic shoulder pain (HSP) after stroke. Two independent authors screened titles and abstracts for the eligibility of the included studies in the electronic databases PubMed and Web of Science. Studies which reported the incidence, prevalence, and risk factors of HSP following stroke were included. The included studies were assessed using the Newcastle-Ottawa Scale for evaluating the quality of nonrandomized studies in meta-analyses. Eighteen studies were included in the final synthesis. In all studies, the number of patients ranged between 58 and 608, with the mean age ranging from 58.7 to 76 years. Seven included studies were rated as "good "quality, while one study rated "fair" and 10 studies rated "poor" quality. Eight studies reported incidence rate while 11 studies reported the prevalence of HSP following a stroke. The incidence of HSP was ranging from 10 to $22 \%$ in the metanalysis of the included studies. The prevalence of HSP was ranging from 22 to $47 \%$ in the metanalysis of the included studies. The most significant predictors of HSP were age, female gender, increased tone, sensory impairment, left-sided hemiparesis, hemorrhagic stroke, hemispatial neglect, positive past medical history, and poor National Institutes of Health Stroke Scale score. The incidence and prevalence of HSP after stroke vary considerably due to various factors. Knowledge of predictors is important to minimize the risk of developing HSP following a stroke.
\end{abstract}

Keywords: stroke; pain; hemiplegic shoulder pain; incidence; prevalence; risk factors

\section{Introduction}

Hemiplegic shoulder pain (HSP) is a common and disabling complication following a stroke, and it may affect the quality of life [1]. It often occurs following two to three months of stroke [2,3]. Consequently, HSP may result in withdrawal from rehabilitation programs, longer hospital stays, reduced limb movement, and impaired quality of life [1]. Numerous causes have been implicated in developing HSP in stroke. This includes muscle flaccidity around the shoulder joint, shoulder subluxation, shoulder-hand syndrome, increased muscle tone, impingement syndrome, frozen shoulder, brachial plexus injury, and the thalamic syndrome $[4,5]$.

There is an inconsistency in reporting incidence and prevalence of HSP following stroke. Some studies reported that the incidence of HSP ranges from $16 \%$ to $84 \%$ [6,7], while others reported a range of $65 \%$ to $70 \%$ [8,9]. In other studies, the incidence rate was ranging from $24 \%$ to $64 \%$ in those stroke populations who are admitted to an inpatient rehabilitation unit [10-13] and it varied from 9\% to $40 \%$ in those who are not admitted [14-16].

A reduced arm motor function at the time of stroke or over a period during rehabilitation was found to be one of the risk factors for developing HSP following stroke [6,10]. Additionally, HSP often occurs and tends to be more severe among people with left-sided hemiplegia $[15,17,18]$. Reduced 
shoulder motion in the affected side of persons with HSP is presented in the initial weeks following stroke [19] and it gradually worsens after one month [19-21]. Other factors probably causing HSP can include shoulder subluxation [22] or rotator cuff injury [23], and those which are related to the neurological problem such as impaired sensation, hemispatial neglect, spasticity, and flaccid paralysis [24,25].

Despite many observational and interventional studies, incidence, prevalence, and the clinical presentations of HSP differ and the exact underlying factors causing HSP are unknown, resulting lack of knowledge in designing effective strategies to prevent and treat HSP. Currently, there were no studies systematically explored the incidence, prevalence, and the risk factors causing shoulder pain in patients with stroke. Therefore, the current systematic review aimed to investigate the incidence, prevalence, and risk factors causing HSP after stroke.

\section{Materials and Methods}

This systematic review followed the guideline for the Meta-analysis Of Observational Studies in Epidemiology (MOOSE) [26]. This systematic review was prospectively registered in PROSPERO (CRD42017077594) and available at http://www.crd.york.ac.uk/PROSPERO/display_record.php?ID= CRD42017077594.

\subsection{Search Strategy}

The electronic databases PubMed, Web of Science, and Scopus were searched until November 19, 2019. Additionally, potential articles were searched manually from the reference list given in each article. The literature search in PubMed was conducted using the following keywords: "Stroke" OR "Hemiplegia") AND ("Shoulder" OR “Arm" OR “Shoulder joint") AND ("Shoulder pain" OR "Pain") AND ("Prevalence" OR "Incidence") [Table 1].

Table 1. Search Strategy.

\begin{tabular}{cccc}
\hline & PubMed & Web of Science & Scopus \\
\hline Date & $19-$ Nov-2019 & $19-N o v-2019$ & $19-N o v-2019$ \\
"Stroke" OR “Hemiplegia" & 291,950 & 296,060 & 395,489 \\
"Shoulder" OR “Arm" OR “Shoulder joint" & 213,412 & 228,005 & 450,679 \\
"Shoulder pain" OR “Pain" & 678,816 & 538,019 & $1,038,105$ \\
"Prevalence" OR “Incidence" & $1,323,666$ & $1,288,045$ & $1,882,741$ \\
Combined search & 94 & 115 & 181 \\
Total minus duplicates & & 139 & \\
\hline
\end{tabular}

\subsection{Eligibility Criteria}

The current review included all the published literature that qualified the following criteria: studies included adults over 18 years of age with a history of stroke for more than one month; the outcomes of included studies should be pain in and around shoulder excluding ribs and the neck pain; all cross-sectional and longitudinal studies to assess the natural course of events after stroke and; studies describing potential risk factors such as demographic factors or impairments related to post-stroke shoulder pain that was evaluated after the incidence of stroke.

Studies were excluded if they were not published in English. Additionally, case reports and case series were also excluded as these types of studies might have a high potential bias. Furthermore, if the cause of shoulder pain was not secondary to stroke, those studies were also excluded (for instance, shoulder pain due to neck pathology).

\subsection{Study Selection}

Two independent authors screened titles and abstract for the eligibility of the included studies. Studies, which reported the incidence, prevalence, and risk factors of HPS following stroke were 
included. Any disagreements between two reviewers in the study selection were discussed and resolved by consensus between them.

\subsection{Data Extraction and Assessment of the Risk of Bias}

Two independent authors (SA and AA) completed the data extraction and assessment of the risk of bias, using structured formats. The important data extraction included the following items: author's name, setting, country of origin, sample size, target population, time since stroke (months), the average age of patients, outcome measurement, and study design. Quality assessment and risk of bias in the selected studies were appraised using the Newcastle-Ottawa Scale for evaluating the quality of nonrandomized studies in meta-analyses $[27,28]$. This evaluation tool has considered three factors (e.g., selection of exposed and non-exposed cohort, comparability of cohorts based on the design or analysis, and outcome based on reliability and validity of the scale, adequate follow-up and dropout rate) to appraise the quality of each included study $[27,28]$. The quality of each study was rated as good, fair, and poor by assigning stars in each domain as per given the guidelines of the Newcastle-Ottawa Scale $[27,28]$. A "good" quality score was given if the included study received 3 or 4 stars in selection domain, 1 or 2 stars in comparability domain, and 2 or 3 stars in outcome domain. A "fair" quality score was given if the included study received 2 stars in selection domain, 1 or 2 stars in comparability domain, and 2 or 3 stars in outcome domain. A "poor" quality score indicated 0 or 1 star(s) in selection domain, or 0 stars in comparability domain, or 0 or 1 star(s) in outcome domain (Table 2). Any disagreements between two reviewers were discussed and resolved by consensus between them.

\subsection{Summary Statistics and Synthesis of Results}

Incidence and prevalence of HSP were reported from the included studies. Prevalence of HSP in the defined group was also reported if the information is available in the included studies. Most significant predictors of HSP after stroke were determined from the included studies. The Comprehensive Meta-Analysis software was used to conduct a meta-analysis. Meta-analysis was conducted for the prevalence, incidence, and risk factors of shoulder pain after stroke if at least two or more included studies with the adequate data for the analysis were available. The event rates for the prevalence and incidence estimates and the odd ratios (ORs) for the risk factors of shoulder pain after stroke were calculated. The effect size with $95 \%$ confidence intervals (CIs) for the prevalence, incidence, and risk factors of shoulder pain after stroke were calculated. 
Table 2. Results of the quality assessment using the Newcastle-Ottawa Quality Assessment Scale criteria.

\begin{tabular}{|c|c|c|c|c|c|c|c|c|c|}
\hline Studies & & Selection & & & Comparability & & Outcome & & Quality Score $†$ \\
\hline & $\begin{array}{l}\text { Representativeness of the exposed } \\
\text { cohort }\end{array}$ & $\begin{array}{l}\text { Selection of the } \\
\text { non-exposed } \\
\text { cohort }\end{array}$ & $\begin{array}{l}\text { Ascertainment of } \\
\text { exposure }\end{array}$ & $\begin{array}{l}\text { Outcome of } \\
\text { Interest Was } \\
\text { Not Present at } \\
\text { Start of Study }\end{array}$ & $\begin{array}{l}\text { Comparability of cohorts } \\
\text { based on the design or analysis }\end{array}$ & $\begin{array}{l}\text { Assessment of } \\
\text { outcome }\end{array}$ & $\begin{array}{c}\text { Follow-Up Long } \\
\text { Enough for } \\
\text { Outcome to Occur } \\
\text { (Median Duration } \\
\text { of Follow-Up } \geq 6 \\
\text { Months) }\end{array}$ & $\begin{array}{l}\text { Adequacy of follow up } \\
\text { of cohorts }\end{array}$ & \\
\hline $\begin{array}{l}\text { Langhorne et al. } \\
\text { (2000) [14] }\end{array}$ & $\begin{array}{l}\text { Three major hospitals in the West of } \\
\text { Scotland were participated. Two } \\
\text { hospitals provided acute stroke } \\
\text { patient care and one hospital } \\
\text { provide acute stroke rehabilitation } \\
\text { care after one-week discharge. } \star\end{array}$ & $\begin{array}{l}\text { No non-exposed } \\
\text { cohort }\end{array}$ & $\begin{array}{l}\text { Weekly assessments in } \\
\text { hospitals by } 3 \text { research } \\
\text { nurses } \star\end{array}$ & Yes $\star$ & $\begin{array}{l}\text { Complication subdivided by } \\
\text { their baseline level of } \\
\text { dependency and compared } \\
\text { using Chi-square test. } \star\end{array}$ & $\begin{array}{c}\text { Questionnaire } \\
\text { based structured } \\
\text { interview } \star\end{array}$ & Yes $\star$ & $\begin{array}{l}100 \% \text { participated at } \\
\text { the 6-month follow-up, } \\
99 \% \text { at the } 18 \text {-month } \\
\text { follow-up, and } 93 \% \text { at } \\
\text { the } 30 \text {-month. } \star\end{array}$ & Good \\
\hline $\begin{array}{l}\text { Gamble et al. } \\
\text { (2000) [29] }\end{array}$ & $\begin{array}{l}\text { Consecutive cohort of stroke patients } \\
\text { admitted to a single hospital. }\end{array}$ & Yes $\star$ & $\begin{array}{l}\text { Patients underwent } \\
\text { interview about } \\
\text { shoulder pain } \star\end{array}$ & Yes $\star$ & $\begin{array}{l}\text { Age, sex, level of anxiety, } \\
\text { disability score, or moderate to } \\
\text { severe motor weakness were } \\
\text { adjusted in chi-square test. } \star\end{array}$ & $\begin{array}{l}\text { Questionnaire } \\
\text { based structured } \\
\text { interview } \star\end{array}$ & No & Not reported & Poor \\
\hline $\begin{array}{l}\text { Gamble et al. } \\
\text { (2002) [16] }\end{array}$ & $\begin{array}{l}\text { Consecutive cohort of stroke patients } \\
\text { admitted to a single hospital. }\end{array}$ & Yes $\star$ & $\begin{array}{l}\text { Patients underwent } \\
\text { interview about } \\
\text { shoulder pain } \star\end{array}$ & Yes $\star$ & $\begin{array}{l}\text { Age, sex, depression and } \\
\text { anxiety scores, and functional } \\
\text { scores were adjusted in } \\
\text { chi-square test. } \star\end{array}$ & $\begin{array}{c}\text { Questionnaire } \\
\text { based structured } \\
\text { interview } \star\end{array}$ & Yes $\star$ & $\begin{array}{l}97 \% \text { participated at the } \\
\text { 6-month follow-up. } \star\end{array}$ & Good \\
\hline $\begin{array}{l}\text { Aras et al. } \\
\text { (2004) [9] }\end{array}$ & $\begin{array}{l}\text { Consecutive cohort of stroke } \\
\text { patients admitted to a single } \\
\text { hospital. }\end{array}$ & Yes $\star$ & $\begin{array}{l}\text { Patients underwent } \\
\text { interview about } \\
\text { shoulder pain } \star\end{array}$ & No & $\begin{array}{l}\text { The presence of spasticity, } \\
\text { thalamic pain, neglect, and } \\
\text { comorbidities were compared } \\
\text { between groups with and } \\
\text { without shoulder pain. } \star\end{array}$ & $\begin{array}{c}\text { Questionnaire } \\
\text { based structured } \\
\text { interview } \star\end{array}$ & No & No statement & Poor \\
\hline $\begin{array}{l}\text { Lindgren et al. } \\
\quad(2007)[6]\end{array}$ & $\begin{array}{l}\text { Participants were representative of } \\
\text { the Lund Stroke Register which } \\
\text { covers the population of Lund-Orup, } \\
\text { including } 8 \text { municipalities } \\
\text { representing the local geographical } \\
\text { area of Lund University Hospital. }\end{array}$ & Yes $\star$ & $\begin{array}{l}\text { Patients underwent } \\
\text { interview about } \\
\text { shoulder pain } \star\end{array}$ & Yes $\star$ & $\begin{array}{l}\text { Univariate analyses were used } \\
\text { to compare arm motor } \\
\text { function, disability, } \\
\text { self-perceived health, } \\
\text { subluxation, and sensory } \\
\text { disturbance, between patients } \\
\text { with and without shoulder } \\
\text { pain. } \star\end{array}$ & $\begin{array}{c}\text { Questionnaire } \\
\text { based structured } \\
\text { interview } \star\end{array}$ & Yes $\star$ & $\begin{array}{l}\text { 79\% participated at the } \\
\text { 4-month follow-up, and } \\
73 \% \text { at the 12-month } \\
\text { follow-up. } \star\end{array}$ & Good \\
\hline $\begin{array}{l}\text { Sackley et al. } \\
(2008)[30]\end{array}$ & $\begin{array}{l}\text { Potential participants were } \\
\text { representative of the Nottingham } \\
\text { Stroke Register, which includes all } \\
\text { stroke admissions to Nottingham } \\
\text { City Hospital and Queens Medical } \\
\text { Centre, Nottingham, UK. }\end{array}$ & $\begin{array}{l}\text { No non-exposed } \\
\text { cohort }\end{array}$ & $\begin{array}{l}\text { Patients underwent } \\
\text { interview about } \\
\text { shoulder pain } \star\end{array}$ & Yes $\star$ & $\begin{array}{l}\text { confounders were not } \\
\text { reported. }\end{array}$ & $\begin{array}{c}\text { Questionnaire } \\
\text { based structured } \\
\text { interview } \star\end{array}$ & Yes $\star$ & $\begin{array}{l}\text { 84\% participated at the } \\
\text { 3-month follow-up, } \\
61 \% \text { at the 6-month } \\
\text { follow-up, and } 50 \% \text { at } \\
\text { the } 2 \text {-month } \\
\text { follow-up. } \star\end{array}$ & Poor \\
\hline $\begin{array}{l}\text { Kuptniratsaikul } \\
\text { et al. (2009) [31] }\end{array}$ & $\begin{array}{l}\text { Participants were representative of } \\
\text { Thai Stroke Rehabilitation Registry, } \\
\text { which maintain the record of } \\
\text { patients with stroke who underwent } \\
\text { rehabilitation } \\
\text { in Thailand. }\end{array}$ & Yes $\star$ & $\begin{array}{l}\text { Patients underwent } \\
\text { interview about } \\
\text { shoulder pain } \star\end{array}$ & No & $\begin{array}{l}\text { Confounders were compared } \\
\text { using the multivariate } \\
\text { analysis. } \star\end{array}$ & $\begin{array}{c}\text { Questionnaire } \\
\text { based structured } \\
\text { interview } \star\end{array}$ & No & No statement & Poor \\
\hline
\end{tabular}


Table 2. Cont.

\begin{tabular}{|c|c|c|c|c|c|c|c|c|c|}
\hline Studies & & Selection & & & Comparability & & Outcome & & Quality Score $†$ \\
\hline $\begin{array}{l}\text { Blennerhassett } \\
\text { et al. (2010) [11] }\end{array}$ & $\begin{array}{l}\text { The } 94 \text { retrospective histories of } \\
\text { patients admitted for inpatient } \\
\text { rehabilitation were audited which } \\
\text { represented } 63 \% \text { of stroke patients in } \\
\text { a 3-year period. }\end{array}$ & Yes $\star$ & Medical report & No & $\begin{array}{l}\text { Confounders were compared } \\
\text { using the logistic regression } \\
\text { analysis. } \star\end{array}$ & $\begin{array}{l}\text { Retrospective } \\
\text { data }\end{array}$ & No & No statement & Poor \\
\hline $\begin{array}{l}\text { Klit et al. (2011) } \\
\text { [32] }\end{array}$ & $\begin{array}{l}\text { Participants were representative of } \\
\text { National Indicator Project database } \\
\text { which records all hospitalized acute } \\
\text { stroke patients in Denmark. } \star\end{array}$ & Yes $\star$ & $\begin{array}{l}\text { Patients reported } \\
\text { shoulder pain }\end{array}$ & Yes $\star$ & $\begin{array}{l}\text { Confounders were compared } \\
\text { using the multiple logistic } \\
\text { regression analysis. }\end{array}$ & $\begin{array}{l}\text { Questionnaire-based } \\
\text { survey }\end{array}$ & No & No statement & Poor \\
\hline $\begin{array}{l}\text { Hansen et al. } \\
\text { (2012) [33] }\end{array}$ & $\begin{array}{l}\text { Consecutive cohort of stroke patients } \\
\text { admitted to a single hospital. }\end{array}$ & No & $\begin{array}{l}\text { Patients underwent a } \\
\text { structured interview. } \\
\star\end{array}$ & No & $\begin{array}{l}\text { Age and gender were adjusted } \\
\text { in chi-square test. } \star\end{array}$ & $\begin{array}{c}\text { Questionnaire } \\
\text { based structured } \\
\text { interview } \star\end{array}$ & Yes $\star$ & $\begin{array}{l}\text { 97\% participated at the } \\
\text { 3-month follow-up, and } \\
92 \% \text { at the 6-month } \\
\text { follow-up. } \\
\star\end{array}$ & Poor \\
\hline $\begin{array}{l}\text { Lindgren et al. } \\
\text { (2012) [34] }\end{array}$ & $\begin{array}{l}\text { Participants were representative of } \\
\text { the Lund Stroke Register which } \\
\text { covers the population of Lund-Orup, } \\
\text { including } 8 \text { municipalities } \\
\text { representing the local geographical } \\
\text { area of Lund University Hospital. } \star\end{array}$ & Yes $\star$ & $\begin{array}{l}\text { Patients underwent } \\
\text { interview about } \\
\text { shoulder pain } \star\end{array}$ & Yes $\star$ & $\begin{array}{l}\text { Univariate analyses were used } \\
\text { to compare age, pain frequency, } \\
\text { affected side, motor function, } \\
\text { and passive range of abduction } \\
\text { between patients with and } \\
\text { without shoulder pain. } \star\end{array}$ & $\begin{array}{l}\text { Questionnaire } \\
\text { based structured } \\
\text { interview } \star\end{array}$ & Yes $\star$ & $\begin{array}{l}\text { 79\% participated at the } \\
\text { 4-month follow-up, and } \\
73 \% \text { at the 12-month } \\
\text { follow-up. } \star\end{array}$ & Good \\
\hline $\begin{array}{l}\text { Kuptniratsaikul } \\
\text { et al. (2013) [35] }\end{array}$ & $\begin{array}{l}\text { Participants were representative of } \\
\text { Thai Stroke Rehabilitation Registry, } \\
\text { which maintain the record of } \\
\text { patients with stroke who underwent } \\
\text { rehabilitation in Thailand. } \star\end{array}$ & Yes $\star$ & $\begin{array}{l}\text { Patients underwent } \\
\text { interview about } \\
\text { shoulder pain } \star\end{array}$ & No & $\begin{array}{l}\text { Confounders were compared } \\
\text { using the multivariate } \\
\text { analysis. } \star\end{array}$ & $\begin{array}{l}\text { Questionnaire } \\
\text { based structured } \\
\text { interview } \star\end{array}$ & Yes $\star$ & $\begin{array}{l}65 \% \text { participated at the } \\
12 \text {-month follow-up. } \star\end{array}$ & Good \\
\hline $\begin{array}{l}\text { Chen et al. } \\
\text { (2014) [36] }\end{array}$ & $\begin{array}{l}\text { The medical records of patients } \\
\text { consecutively admitted to a } \\
\text { single hospital. }\end{array}$ & No & Retrospective data & No & $\begin{array}{l}\text { Confounders were compared } \\
\text { using the Chi-square test. } \star\end{array}$ & $\begin{array}{l}\text { Retrospective } \\
\text { medical } \\
\text { record data }\end{array}$ & No & No statement & Poor \\
\hline
\end{tabular}


Table 2. Cont.

\begin{tabular}{|c|c|c|c|c|c|c|c|c|c|}
\hline Studies & & Selection & & & Comparability & & Outcome & & Quality Score + \\
\hline $\begin{array}{c}\text { Kim et al. (2014) } \\
\text { [37] }\end{array}$ & $\begin{array}{l}\text { Consecutive cohort of stroke patients } \\
\text { admitted to a single hospital. }\end{array}$ & Yes $\star$ & $\begin{array}{l}\text { Patients underwent } \\
\text { interview about } \\
\text { shoulder pain } \star\end{array}$ & Yes $\star$ & $\begin{array}{l}\text { Age, gender and significant } \\
\text { variables from the univariate } \\
\text { analysis were included in the } \\
\text { final multivariate logistic } \\
\text { regression model. } \star\end{array}$ & $\begin{array}{l}\text { Questionnaire } \\
\text { based structured } \\
\text { interview } \star\end{array}$ & Yes $\star$ & $\begin{array}{l}78 \% \text { participated at the } \\
\text { 3-month follow-up, and } \\
62 \% \text { at the 6-month } \\
\text { follow-up. } \star\end{array}$ & Good \\
\hline $\begin{array}{l}\text { Kwon et al. } \\
\text { (2014) [38] }\end{array}$ & $\begin{array}{l}\text { Participants were representative of } \\
\text { eight rehabilititation units situated in } \\
\text { three different large local catchment } \\
\text { area in the Republic of Korea. } \star\end{array}$ & Yes $\star$ & $\begin{array}{l}\text { Patients underwent } \\
\text { interview about } \\
\text { shoulder pain } \star\end{array}$ & No & $\begin{array}{l}\text { Age, sex, the Motricity Index of } \\
\text { the upper and lower limbs, } \\
\text { and ambulatory types were } \\
\text { included in the multivariate } \\
\text { logistic regression model. } \star\end{array}$ & $\begin{array}{c}\text { Questionnaire } \\
\text { based structured } \\
\text { interview } \star\end{array}$ & No & No statement & Poor \\
\hline $\begin{array}{l}\text { Karaahmet et al. } \\
\quad(2014)[39]\end{array}$ & $\begin{array}{l}\text { Consecutive cohort of stroke patients } \\
\text { admitted to a single physical } \\
\text { medicine and rehabilitation clinic. }\end{array}$ & Yes $\star$ & $\begin{array}{l}\text { Patients underwent } \\
\text { interview about } \\
\text { shoulder pain } \star\end{array}$ & No & $\begin{array}{c}\text { Disease duration, neglect, } \\
\text { sensory disturbance, spasticity, } \\
\text { immobilization, late } \\
\text { rehabilitation, and motor } \\
\text { function were included in } \\
\text { backward stepwise } \\
\text { multinomial } \\
\text { logistic regression analysis. } \star\end{array}$ & $\begin{array}{c}\text { Questionnaire } \\
\text { based structured } \\
\text { interview } \star\end{array}$ & Yes $\star$ & No statement & Fair \\
\hline $\begin{array}{l}\text { Adey-Wakeling } \\
\text { et al. (2015) [3] }\end{array}$ & $\begin{array}{l}\text { A population-based stroke incidence } \\
\text { study conducted in a specific region } \\
\text { of the western suburbs of Adelaide, } \\
\text { South Australia. } \star\end{array}$ & Yes $\star$ & $\begin{array}{l}\text { Patients underwent } \\
\text { interview about } \\
\text { shoulder pain } \star\end{array}$ & No & $\begin{array}{l}\text { logistic regression models were } \\
\text { used to analyses different } \\
\text { confounders. } \star\end{array}$ & $\begin{array}{c}\text { Questionnaire } \\
\text { based structured } \\
\text { interview } \star\end{array}$ & Yes $\star$ & $\begin{array}{l}\text { 78\% participated at the } \\
\text { 4-month follow-up, and } \\
75 \% \text { at the } 12 \text {-month } \\
\text { follow-up. } \star\end{array}$ & Good \\
\hline $\begin{array}{l}\text { Paolucci et al. } \\
(2016)[40]\end{array}$ & $\begin{array}{c}\text { Consecutive cohort of stroke } \\
\text { patients admitted to at eight Italian } \\
\text { hospitals. } \star\end{array}$ & No & $\begin{array}{l}\text { Patients underwent } \\
\text { interview about } \\
\text { shoulder pain } \star\end{array}$ & No & $\begin{array}{l}\text { Age, gender, type of stroke, } \\
\text { and severity of stroke were } \\
\text { included in regression } \\
\text { analysis. } \star\end{array}$ & $\begin{array}{l}\text { Questionnaire } \\
\text { based structured } \\
\text { interview } \star\end{array}$ & No & No statement & Poor \\
\hline
\end{tabular}

† Good quality: 3 or 4 stars $(\star)$ in selection domain AND 1 or 2 stars in comparability domain AND 2 or 3 stars in outcome domain; Fair quality: 2 stars in selection domain AND 1 or 2 stars in comparability domain AND 2 or 3 stars in outcome/exposure domain; Poor quality: 0 or 1 star in selection domain OR 0 stars in comparability domain OR 0 or 1 stars in outcome/exposure domain. 


\section{Results}

\subsection{Study Selection}

Based on the abstract and title search, initially, 390 articles were identified. After excluding duplicates $(N=251)$ and screening of abstracts, a total of 330 studies were not relevant to the current review, therefore excluded. Sixty full-text articles were included in the final screening, of which 42 articles failed to match the inclusion criteria, and were hence excluded. Henceforth, a total of 18 studies included in the final synthesis $[3,6,9,11,14,16,28-39]$. Figure 1 shows the details of the study selection process and results of the literature search [26].

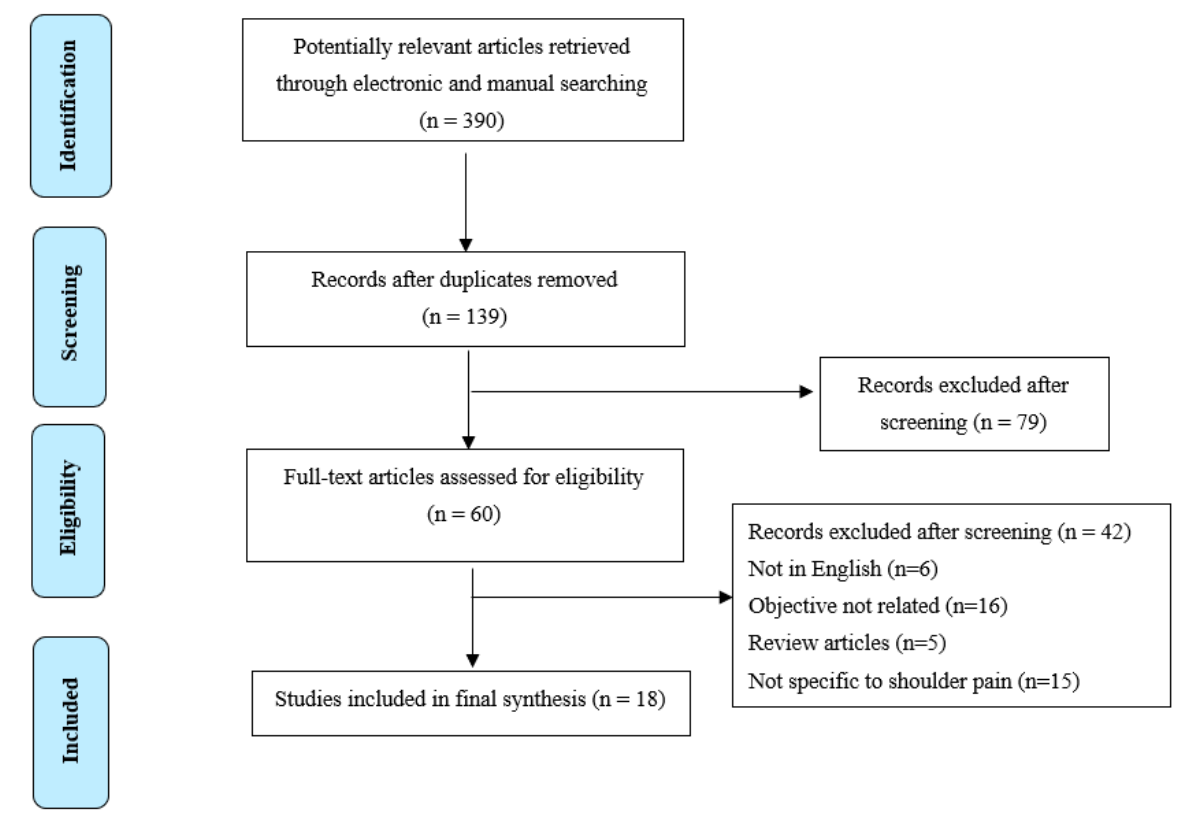

Figure 1. Study selection process and results of the literature search (PRISMA flow diagram).

\subsection{Characteristics of Included Studies}

Table 3 present the characteristics of included studies. Among 18 included studies [3,6,9,11, 14,16,29-40], sixteen were categorized as a prospective observational study $[3,6,9,14,16,29-35,37-40]$, while others as a retrospective observational study [11,36]. Studies originated from the UK [14,16, 29,30], Turkey [9,39], Sweden [6,34], Thailand [31,35], Australia [3,11], Denmark [32,33], Taiwan [36], Korea [37,38], and Italy [40]. In all studies, the number of patients ranged between 58 and 608 with the mean age ranging from 58.7 to 76 years. Four included studies have defined participants as first-ever stroke patients $[6,34,36,37]$. Most of the other studies have defined participants as patients with stroke $[3,11,14,31-33,35,38-40]$, while two studies [16,29] have defined participants as patients with a diagnosis of acute stroke. In all studies, the time since incidence of a stroke at recruitment was within one week of symptom onset up to 30 months after stroke. Seven include studies used a visual analog scale (VAS) [3,6,14,16,29,30,34], two studies used a numerical rating scale (NRS) [32,37], one study used a Neuropathic Pain Symptom Inventory [40], one study used the University of Alabama's Pain Behaviors Scale [38], one study used medical records [36], and one study used an interview method [33], while others used physical examination to assess shoulder pain after stroke $[9,11,31,35,39]$. 
Table 3. Study characteristics.

\begin{tabular}{|c|c|c|c|c|c|c|c|}
\hline & Setting/Country & Sample Size & Targeted Population & $\begin{array}{l}\text { Time since Stroke } \\
\text { (Months) at } \\
\text { Recruitment }\end{array}$ & $\begin{array}{l}\text { Average Age at } \\
\text { Recruitment }\end{array}$ & Outcome Measurement & Design \\
\hline Langhorne et al. (2000) [14] & $\begin{array}{l}\text { Multicenter hospital-based } \\
\text { study/Scotland, UK. }\end{array}$ & $N=311$ & $\begin{array}{l}\text { People with stroke, } \\
\text { Hemiplegia }\end{array}$ & $\begin{array}{l}\text { up to } 30 \text { months after } \\
\text { stroke }\end{array}$ & $\begin{array}{l}76 \text { years (interquartile } \\
\text { range } 70 \text { to } 82 \text { years) }\end{array}$ & VAS & A prospective study \\
\hline Gamble et al. (2000) [29] & Hospital-based study/UK & $N=123$ & $\begin{array}{l}\text { Patients with a } \\
\text { diagnosis of acute } \\
\text { stroke }\end{array}$ & up to 6 months & 70.6 years & VAS & A prospective study \\
\hline Gamble et al. (2002) [16] & Hospital-based study/UK & $N=123$ & $\begin{array}{l}\text { Patients with a } \\
\text { diagnosis of acute } \\
\text { stroke }\end{array}$ & up to 6 months & 70.6 years (range 29-93) & VAS & A prospective study \\
\hline Aras et al. (2004) [9] & $\begin{array}{l}\text { Hospital-based } \\
\text { study/Turkey }\end{array}$ & $N=85$ & $\begin{array}{l}\text { Patients with } \\
\text { hemiplegia }\end{array}$ & $\begin{array}{l}64.8 \text { days from the } \\
\text { onset }\end{array}$ & 58.7 years & Physical examination & A prospective study \\
\hline Lindgren et al. (2007) [6] & $\begin{array}{l}\text { Population-Based } \\
\text { Study/Sweden }\end{array}$ & $N=416$ & $\begin{array}{c}\text { First-ever stroke } \\
\text { patients }\end{array}$ & up to 16 months & $\begin{array}{c}73.1 \text { years (range 17-102 } \\
\text { years) }\end{array}$ & VAS & A prospective study \\
\hline Sackley et al. (2008) [30] & Hospital-based study/UK & $N=600$ & 3-months post stroke & $\begin{array}{l}\text { 3-months from the } \\
\text { onset up to } 12 \text {-months }\end{array}$ & $\begin{array}{c}76 \text { years (range 31-98 } \\
\text { years) }\end{array}$ & VAS & A prospective study \\
\hline Kuptniratsaikul et al. (2009) [31] & $\begin{array}{l}\text { Multicenter hospital-based } \\
\text { study/Thailand }\end{array}$ & $N=327$ & Patients with stroke & more than two months & 62.2 years (SD 12.1) & Physical examination & A prospective study \\
\hline Blennerhassett et al. (2010) [11] & $\begin{array}{c}\text { Hospital-based } \\
\text { data/Australia }\end{array}$ & $N=94$ & Patients with stroke & More than 2 months & $\begin{array}{c}59 \text { years (range } 17-80 \\
\text { years) }\end{array}$ & Physical examination & $\begin{array}{l}\text { Retrospective observational } \\
\text { study }\end{array}$ \\
\hline Klit et al. (2011) [32] & $\begin{array}{l}\text { Population-based } \\
\text { study/Denmark }\end{array}$ & $\begin{array}{c}N=608 \text { (stroke } \\
\text { patients), } 519 \\
\text { (reference subjects) }\end{array}$ & Patients with stroke & $\begin{array}{l}\text { Median days from } \\
\text { stroke 794.5(range } \\
588-1099)\end{array}$ & Median age, 72.6 years & NRS & A prospective study \\
\hline Lindgren et al. (2012) [34] & $\begin{array}{l}\text { Hospital-based } \\
\text { study/Sweden }\end{array}$ & $N=58$ & $\begin{array}{c}\text { First-ever stroke } \\
\text { patients }\end{array}$ & up to 16 months & 71 years & VAS & A prospective study \\
\hline Kuptniratsaikul et al. (2013) [35] & $\begin{array}{l}\text { Multicenter hospital-based } \\
\text { study/Thailand }\end{array}$ & $N=327$ & Patients with stroke & 12 months of onset & 62.1 years (SD 12.5 years) & Physical examination & A prospective study \\
\hline Chen et al. (2014) [36] & $\begin{array}{c}\text { Hospital-based } \\
\text { study/Taiwan }\end{array}$ & $N=568$ & $\begin{array}{l}\text { First-time stroke } \\
\text { patients }\end{array}$ & Not reported & 65.7 years (SD 13.3 years) & Medical records & $\begin{array}{l}\text { A retrospective longitudinal } \\
\text { cohort study }\end{array}$ \\
\hline Kim et al. (2014) [37] & Hospital-based study/Korea & $N=94$ & $\begin{array}{l}\text { Patients with first-ever } \\
\text { unilateral stroke lesion }\end{array}$ & $\begin{array}{l}\text { up to } 6 \text { months } \\
\text { post-stroke }\end{array}$ & 65.6 years & NRS & A prospective study \\
\hline Kwon et al. (2014) [38] & Hospital-based study/Korea & $N=229$ & Patients with stroke & More than 2 months & 59.0 years (SD 12.4) & $\begin{array}{l}\text { University of Alabama's } \\
\text { Pain Behaviors Scale }\end{array}$ & A prospective study \\
\hline Karaahmet et al. (2014) [39] & $\begin{array}{l}\text { Hospital-based } \\
\text { study/Turkey }\end{array}$ & $N=63$ & Patients with stroke & More than 2 months & $\begin{array}{l}61 \text { years (range, } \\
39-85 \text { years) }\end{array}$ & Physical examination & A prospective study \\
\hline Adey-Wakeling et al. (2015) [3] & $\begin{array}{l}\text { Population-Based } \\
\text { Study/Australia }\end{array}$ & $N=318$ & Patients with stroke & $\begin{array}{l}\text { Average } 8.7 \text { days post } \\
\text { onset up to } 12 \text { years }\end{array}$ & 72.5 years & VAS & A prospective study \\
\hline Paolucci et al. (2016) [40] & $\begin{array}{l}\text { Hospital-based multicenter } \\
\text { study/Italy }\end{array}$ & $N=443$ & Patients with stroke & $\begin{array}{l}\text { more than } 90 \text { days } \\
\text { onset of stroke }\end{array}$ & 67.1 years & $\begin{array}{l}\text { Neuropathic Pain Symptom } \\
\text { Inventory }\end{array}$ & A prospective study \\
\hline
\end{tabular}

VAS: Visual analogue scale; NRS: Numerical rating scale. 


\subsection{Methodological Quality}

Table 2 presented the results of the quality assessment using the Newcastle-Ottawa Scale for evaluating the quality of nonrandomized studies in meta-analyses $[27,28]$. Seven included studies [3,6,14,16,34,35,37] were rated as "good "quality, while one study [39] rated "fair" and 10 studies $[9,11,29-33,36,38,40]$ rated "poor" quality. Five included studies $[14,30,33,36,40]$ did not have any non-exposed control group. More than half of the included studies $[3,9,11,31,33,35,36,38-40]$ had reported the presence of outcome at the start of the study. Eight included studies $[9,11,29,31,32,36,38,40]$ reported less than 6-month of follow-up. Nine included studies $[9,11,29,31,32,36,38-40]$ did not provide information about the dropouts.

\subsection{Incidence and Prevalence of HSP after Stroke}

The details of the incidence and prevalence of HSP after stroke is given in Table 4. The overall incidence of HSP was ranging from 1.6 to $40 \%$ in the included studies. The overall prevalence of HSP was ranging from 9.41 to $91.9 \%$ in the included studies. The incidence of HSP after a stroke at admission was reported in two studies [3,14]. Incidence at admission ranged from 9 to $10 \%$. Three studies reported on the prevalence of HSP after a stroke at admission $[9,11,39]$, ranging from 23 to $63.5 \%$. The incidence of HSP after a stroke at 6 months was reported in three studies [14,16,33], ranging from 15 to $40 \%$. Only one study reported the 6-month prevalence (42\%) of HSP after a stroke [30]. Only one study reported the 12-month incidence (21\%) of HSP after stroke [3]. Two studies reported the 12-month prevalence of HSP after stroke [30,35], ranging from 8.5 to $47 \%$. Only one study reported the two-year incidence (15.1\%) of HSP after a stroke [32]. 
Table 4. Incidence and prevalence of shoulder pain after stroke in the included studies.

\begin{tabular}{|c|c|c|c|}
\hline Study & Incidence [Proportion (95\% CI)] & Prevalence [Proportion $(95 \% \mathrm{CI})]$ & Prevalence in Defined Group \\
\hline $\mathrm{L}$ & $\begin{array}{l}\text { Incidence at admission: 9\% (6-12\%) } \\
\text { Discharge to 6-month incidence: } 15 \%(9-21 \%) \\
\text { 6-months to 18-months incidence: } 11 \%(6-16 \%) \\
\text { 18-months to 30-months incidence: } 12 \%(6-17 \%)\end{array}$ & Weekly point prevalence: $6 \%(5-7 \%)$ & \\
\hline Gamble et al. (2000) [29] & Incidence at 2-week: $25 \%$ & & \\
\hline Gamble et al. (2002) [16] & Incidence at 6-months: $40 \%$ & & \\
\hline Aras et al. (2004) [9] & & Prevalence at admission: $63.5 \%$ & \\
\hline Lindgren et al. (2007) [6] & & $\begin{array}{l}\text { Prevalence at } 4 \text {-months: } 22 \% \\
\text { Prevalence at 16-months: } 24 \%\end{array}$ & $\begin{array}{l}\text { Functional status independence: } 37 \% \\
\text { Moderate dependence: } 31 \% \\
\text { Major dependence: } 32 \% \\
\text { Self-perceived ill health: } 23 \% \\
\text { Arm motor function } \\
\text { No function: } 27 \% \\
\text { Reduced function: } 56 \% \\
\text { Normal function: } 17 \% \\
\text { Sensory disturbance for light touch: } 31 \% \\
\text { Shoulder Subluxation: } 41 \%\end{array}$ \\
\hline Sackley et al. (2008) [30] & & $\begin{array}{l}\text { Overall prevalence: } 52 \% \\
\text { Prevalence at 3-months: } 36 \% \\
\text { Prevalence at 6-months: } 42 \% \\
\text { Prevalence at 12-months: } 47 \%\end{array}$ & \\
\hline Kuptniratsaikul et al. (2009) [31] & & Overall prevalence: $19 \%$ & $\begin{array}{l}\text { Hemorrhagic stroke, prevalence of shoulder pain: } 26.1 \% \\
\text { Infarction stroke, prevalence of shoulder pain: } 16.2 \%\end{array}$ \\
\hline Blennerhassett et al. (2010) [11] & Incidence during inpatient: 11.7\% & $\begin{array}{l}\text { Prevalence at admission: } 23 \% \\
\text { Prevalence during inpatient: } 35 \%\end{array}$ & \\
\hline Klit et al. (2011) [32] & Two-year incidence: $15.1 \%$ & & \\
\hline Hansen et al. (2012) [33] & $\begin{array}{l}\text { Incident at onset: } 1.5 \% \\
\text { Incident at 3-months: } 13.1 \% \\
\text { Incident at 6-months: } 16.4 \%\end{array}$ & & $\begin{array}{l}\text { Shoulder pain in stroke-affected side at onset: } 1.1 \% \\
\text { at 3-months: } 10.2 \% \\
\text { at 6-months: } 12.0 \%\end{array}$ \\
\hline Lindgren et al. (2012) [34] & & $\begin{array}{l}\text { Prevalence at } 4 \text { and } 16 \text {-months: } 19 \% \text { and } 72 \% \text {, } \\
\text { respectively }\end{array}$ & \\
\hline Kuptniratsaikul et al. (2013) [35] & & Prevalence at 12 -months: $8.5 \%$ & \\
\hline Chen et al. (2014) [36] & $\begin{array}{l}\text { Incidence in acute ward: } 2.6 \% \\
\text { Incidence in rehabilitation ward:23.2\% }\end{array}$ & & $\begin{array}{l}\text { Incidence in rehabilitation ward (age group wise) } \\
<65 \text { years: } 23.4 \% \\
65-75 \text { years: } 22.1 \% \\
\geq 75 \text { years: } 24.5 \%\end{array}$ \\
\hline
\end{tabular}


Table 4. Cont.

\begin{tabular}{|c|c|c|c|}
\hline Study & Incidence [Proportion $(95 \% \mathrm{CI})]$ & Prevalence [Proportion $(95 \% \mathrm{CI})]$ & Prevalence in Defined Group \\
\hline Kim et al. (2014) [37] & Not reported & Not reported & Not reported \\
\hline Kwon et al. (2014) [38] & & Overall prevalence: $91.9 \%$ & $\begin{array}{l}\text { Prevalence of shoulder pain based on ambulatory mode } \\
\text { Independent: } 93.3 \% \\
\text { Cane: } 89.2 \% \\
\text { Wheelchair: } 70 \%\end{array}$ \\
\hline Karaahmet et al. (2014) [39] & & $\begin{array}{l}\text { Prevalence at admission: } 53 \% \\
\text { Prevalence at discharge: } 62 \%\end{array}$ & $\begin{array}{l}\text { Prevalence of HSP with other complications } \\
\text { Neglect: } 90 \% \\
\text { Aphasia: } 55.6 \% \\
\text { Depression: } 65 \% \\
\text { Spasticity: } 78.9 \% \\
\text { Sensory disturbance: } 40 \% \\
\text { Subluxation: } 77.8 \%\end{array}$ \\
\hline Adey-Wakeling et al. (2015) [3] & $\begin{array}{l}\text { Incidence at admission: } 10 \% \\
\text { Incidence at } 4 \text { months: } 21 \% \\
\text { Incidence at } 12 \text { months: } 21 \% \\
\text { Overall incidence: } 29 \%\end{array}$ & & $\begin{array}{l}\text { Female: } 46 \% \\
\text { Medical history } \\
\text { Previous stroke: } 12 \% \\
\text { Previous MI: } 17 \% \\
\text { Hypertension: } 71 \% \\
\text { Diabetes: } 28 \% \\
\text { History of shoulder pain: } 27 \% \\
\text { Stroke subbtype } \\
\text { Total ischemic: } 88 \% \\
\text { Large artery: } 14 \% \\
\text { Cardio embolic: } 34 \% \\
\text { Lacunar: } 9 \% \\
\text { Other/unknown ischemic: } 32 \% \\
\text { Hemorrhagic: } 9 \% \\
\text { Unknown: } 2 \% \\
\text { Left side: } 52 \% \\
\text { High NIHSS score (> median): } 5 \% \\
\text { Motor arm } \\
\text { Reduced function: } 38 \% \\
\text { No function: } 31 \%\end{array}$ \\
\hline Paolucci et al. (2016) [40] & & $\begin{array}{l}\text { Overall mean prevalence: } 9.41 \% \\
\text { Acute phase prevalence: } 0.63 \% \\
\text { Sub-acute phase prevalence: } 17.27 \% \\
\text { Chronic phase prevalence: } 10.34 \%\end{array}$ & \\
\hline
\end{tabular}




\subsection{Risk Factors of HSP after Stroke}

Table 5 presented the risk factors of HSP used in the included studies. A variety of risk factors was assessed in the included studies $[3,6,9,11,14,16,29,31,32,34,37,39]$. The most significant risk factors of HSP were age, arm weakness, sensory impairment, abnormal shoulder joint examination, average depression score, functional status, self-perceived ill health, subluxation, pathogenesis, left-sided hemiparesis, and prior history of shoulder pain. Six studies did not assess the risk factors of HSP after stroke $[30,33,35,36,38,40]$.

Table 5. Shoulder pain and risk factors used in the included studies.

\begin{tabular}{|c|c|c|}
\hline Study & $\begin{array}{c}\text { Risk Factors of Shoulder Pain Which Were } \\
\text { Assessed }\end{array}$ & $\begin{array}{c}\text { Odd Ratios (OR) [95\% Confidence } \\
\text { Interval (CI)] }\end{array}$ \\
\hline \multirow{10}{*}{ Blennerhassett et al. (2010) [11] } & Gender (male) & $0.89(0.36$ to 2.16$)$ \\
\hline & Altered Tone & $18.94(6.46$ to 55.51$)$ \\
\hline & Subluxation & $19.34(5.57$ to 65.94$)$ \\
\hline & Sensory deficits & 2.59 (1.08 to 6.17$)$ \\
\hline & Inattention/neglect & $1.53(0.59$ to 3.97$)$ \\
\hline & Cognitive impairment & 1.03 (0.44 to 2.40$)$ \\
\hline & Impaired communication & $1.48(0.62$ to 3.50$)$ \\
\hline & Type of stroke & $0.76(0.26$ to 2.17$)$ \\
\hline & Hand dominance & 0.24 (0.03 to 2.05$)$ \\
\hline & Previous shoulder problem & 2.55 (0.63 to 10.22$)$ \\
\hline \multirow{7}{*}{ Klit et al. (2011) [32] } & Males (vs. females) & $0.77(0.53-1.11)$ \\
\hline & Age $65-74$ years (vs. < 65 years) & $0.57(0.36-0.90)$ \\
\hline & Age $>75$ years (vs. $<65$ years) & $0.65(0.43-0.99)$ \\
\hline & Diabetes (vs. no diabetes) & $1.08(0.65-1.78)$ \\
\hline & Depression (vs. no depression) & $3.43(2.25-5.25)$ \\
\hline & Infarction (vs. hemorrhage) & $0.73(0.43-1.26)$ \\
\hline & Unspecified (vs. hemorrhage) & $1.09(0.57-2.09)$ \\
\hline \multirow{4}{*}{ Lindgren et al. (2012) [34] } & Left-sided hemiparesis & $10.47(1.92-57.05), p=0.01$ \\
\hline & Pain frequency & $6.85(1.46-32.14), p=0.02$ \\
\hline & Decreased passive abduction & $4.46(0.99-20.10), p=0.05$ \\
\hline & Age & $1.05(0.99-1.12), p=0.07$ \\
\hline \multirow{5}{*}{ Kim et al. (2014) [37] } & Young age $(<70$ years $)$ & $3.65(1.250-10.637), p=0.018$ \\
\hline & Male & $0.99(0.370-2.683), p=0.99$ \\
\hline & Poor NIHSS item 5 score $(\geq 3)$ & $2.96(1.141-7.665), p=0.026$ \\
\hline & $\begin{array}{l}\text { Presence of long head of biceps } \\
\text { tendon effusion }\end{array}$ & $2.35(0.897-6.150), p=0.082$ \\
\hline & $\begin{array}{l}\text { Presence of supraspinatus tendon } \\
\text { tendinosis/tear }\end{array}$ & $4.21(1.372-12.931), p=0.012$ \\
\hline \multirow{10}{*}{ Karaahmet et al. (2014) [39] } & Neglect & $7.20(0.840-61.4690), p=0.071$ \\
\hline & Sensory disturbance & $3.26(0.478-22.301), p=0.228$ \\
\hline & Spasticity & $1.56(0.272-9.002), p=0.617$ \\
\hline & Immobilization & $3.28(0.527-20.457), p=0.203$ \\
\hline & Late rehabilitation & $0.52(0.025-10.658), p=0.669$ \\
\hline & Disease duration & $1.05(0.964-1.134), p=0.279$ \\
\hline & Baseline FMA (Fugl-Meyer Motor Assessment.) & $0.99(0.905-1.083), p=0.822$ \\
\hline & Baseline FAT (Frenchay Arm Test) & \\
\hline & Baseline FIM (Functional Independence Measure) & $0.66(0.234-1.872), p=0.437$ \\
\hline & & $1.01(0.970-1.045), p=0.720$ \\
\hline \multirow{19}{*}{ Adey-Wakeling et al. (2015) [3] } & Mean age (y) & $0.96(0.79-1.17), p=0.690$ \\
\hline & Sex: female & $1.03(0.77-1.37), p=0.845$ \\
\hline & Medical history & \\
\hline & Previous stroke & $0.47(0.21-1.07), p=0.074$ \\
\hline & Previous MI & $1.16(0.53-2.54), p=0.705$ \\
\hline & Hypertension & $0.96(0.50-1.85), p=0.907$ \\
\hline & Diabetes & $1.20(0.62-2.30), p=0.587$ \\
\hline & History of shoulder pain & $8.09(3.16-20.75), p=<0.0001$ \\
\hline & Stroke subtype & \\
\hline & Cardioembolic & $1.10(0.60-2.01), p=0.767$ \\
\hline & Lacunar & $0.85(0.36-2.04), p=0.719$ \\
\hline & Other/unknown ischemic & $1.45(0.78-2.72), p=0.241$ \\
\hline & Hemorrhagic & $1.22(0.49-3.01), p=0.670$ \\
\hline & Unknown & $0.57(0.09-3.47), p=0.541$ \\
\hline & Right Hemiparesis & $0.87(0.65-1.17), p=0.350$ \\
\hline & High NIHSS score (> median) & $1.39(0.78-2.49), p=0.268$ \\
\hline & Motor arm & \\
\hline & Reduced function & $1.20(0.79-1.83), p=0.399$ \\
\hline & No function & $1.91(1.20-3.04), p=0.007$ \\
\hline
\end{tabular}

\subsection{Meta-Analysis}

Of the 10 included studies with adequate prevalence data, three estimated event rates for the prevalence at admission, two estimated event rates for the prevalence at 4-month, 12-month, and 
16-month, while four estimated event rates for an overall prevalence of shoulder pain after stroke. Figure 2 indicates a forest plot of prevalence rates with effect sizes and 95\% CIs. Of the five included studies with adequate incidence data, two estimated event rates of the incidence at admission, while three estimated event rates of incidence at 6-month of shoulder pain after stroke. Figure 3 indicates a forest plot of incidence rates with effect sizes and $95 \%$ CIs. Of the eight included studies with adequate risk estimate data, three studies estimated ORs stratified by gender, two stratified by tone, sensation, affected body side, hemispatial neglect, and National Institutes of Health Stroke Scale Score, while three stratified based on type of strokes and seven stratified based on past medical history. Figures 4 and 5 indicate a forest plot of risk factors with effect sizes and 95\% CIs.

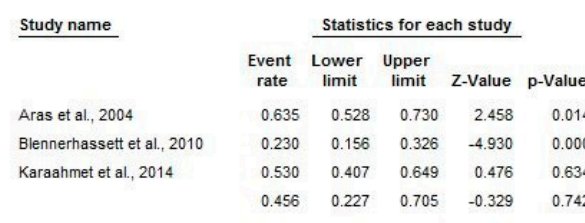

A. Prevalence at admission

\begin{tabular}{lccccr} 
Study name & & \multicolumn{3}{c}{ Statistics for each study } \\
\cline { 3 - 6 } & $\begin{array}{c}\text { Event } \\
\text { rate }\end{array}$ & $\begin{array}{c}\text { Lower } \\
\text { limit }\end{array}$ & $\begin{array}{c}\text { Upper } \\
\text { limit }\end{array}$ & Z-Value & p-Value \\
Lndgren et al., 2007 & 0.220 & 0.183 & 0.262 & -10.694 & 0.000 \\
Lndgren et al., 2012 & 0.190 & 0.109 & 0.311 & -4.332 & 0.000 \\
& 0.217 & 0.182 & 0.256 & -11.526 & 0.000
\end{tabular}

\section{Event rate and $95 \% \mathrm{Cl}$}

0.000
.000
0.000

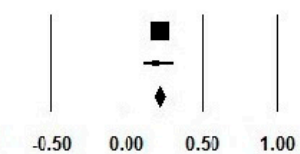

B. Prevalence at 4-month
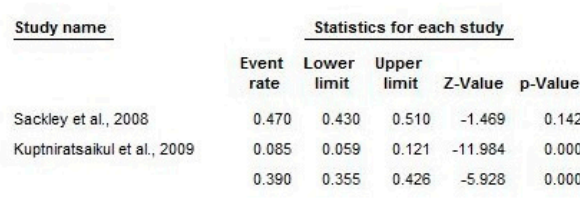

Event rate and $95 \% \mathrm{Cl}$ rate limit limit $\mathrm{z}$-Value $\mathrm{p}$-Value $\begin{array}{lllll}0.470 & 0.430 & 0.510 & -1.469 & 0.142\end{array}$ $\begin{array}{lllll}0.390 & 0.355 & 0.426 & -5.928 & 0.000\end{array}$

C. Prevalence at 12-month

\begin{tabular}{lcccrrr} 
Study name & \multicolumn{5}{c}{ Statistics for each study } \\
\cline { 3 - 6 } & $\begin{array}{c}\text { Event } \\
\text { rate }\end{array}$ & $\begin{array}{c}\text { Lower } \\
\text { limit }\end{array}$ & $\begin{array}{c}\text { Upper } \\
\text { limit }\end{array}$ & Z-Value & p-Value \\
Lindgren et al., 2007 & 0.240 & 0.201 & 0.283 & -10.041 & 0.000 \\
Lindgren et al., 2012 & 0.720 & 0.592 & 0.820 & 3.230 & 0.001 \\
& 0.470 & 0.102 & 0.874 & -0.116 & 0.908
\end{tabular}

D. Prevalence at 16 -month

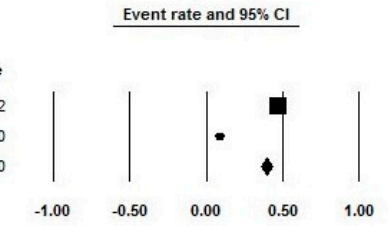

$\underline{\text { Study name }}$

Statistics for each study

Event Lower Upper

rate limit limit Z-Value $p$-Value

Sackley et al., 2008

$\begin{array}{lllll}0.520 & 0.480 & 0.560 & 0.980 & 0.327\end{array}$

$\begin{array}{llllll}\text { Kuplniralsaikul el al., } 2009 & 0.190 & 0.151 & 0.236 & -10.286 & 0.000\end{array}$

$\begin{array}{llllll}\text { Kwon et al., } 2014 & 0.920 & 0.877 & 0.949 & 10.027 & 0.000\end{array}$

Paolucci et al., 2016

$\begin{array}{rrrrr}0.920 & 0.877 & 0.949 & 10.027 & 0.000 \\ 0.094 & 0.070 & 0.125 & -13.917 & 0.000\end{array}$

$\begin{array}{lllll}0.424 & 0.139 & 0.770 & -0.398 & 0.691\end{array}$

Event rate and $95 \% \mathrm{Cl}$

E. Overall Prevalence

Figure 2. Prevalence of shoulder pain after stroke ((A). Prevalence at admission, (B). Prevalence at 4-month, (C). Prevalence at 12-month, (D). Prevalence at 16-month, (E). Overall prevalence). 
$\underline{\text { Study name }}$

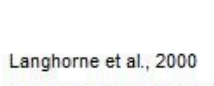
Adey-Wakeling et al., 2015

\begin{tabular}{cccccc} 
& \multicolumn{3}{c}{ Statistics for each study } & \\
\cline { 2 - 5 } $\begin{array}{c}\text { Event } \\
\text { rate }\end{array}$ & $\begin{array}{c}\text { Lower } \\
\text { limit }\end{array}$ & $\begin{array}{c}\text { Upper } \\
\text { limit }\end{array}$ & Z-Value & p-Value \\
0.090 & 0.063 & 0.127 & -11.677 & 0.000 \\
0.100 & 0.072 & 0.138 & -11.755 & 0.000 \\
0.095 & 0.075 & 0.121 & -16.563 & 0.000
\end{tabular}

A. Incidence at admission

Event rate and $95 \% \mathrm{Cl}$

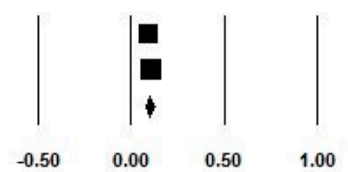

Event rate and $95 \% \mathrm{Cl}$

Study name

Langhorne et al., 2000
Gamble et al., 2002
Hansen et al., 2012

Statistics for each study

$\begin{array}{cccr}\begin{array}{c}\text { Event } \\ \text { rate }\end{array} & \begin{array}{c}\text { Lower } \\ \text { limit }\end{array} & \begin{array}{c}\text { Upper } \\ \text { limit }\end{array} & \text { Z-Value } \\ 0.150 & 0.114 & 0.194 & -10.923 \\ 0.400 & 0.317 & 0.489 & -2.203 \\ 0.164 & 0.126 & 0.210 & -10.428 \\ 0.221 & 0.114 & 0.384 & -3.132\end{array}$

\section{B. Incidence at 6-month}

Figure 3. Incidence of shoulder pain after stroke ((A). Incidence at admission, (B). Incidence at 6-month).

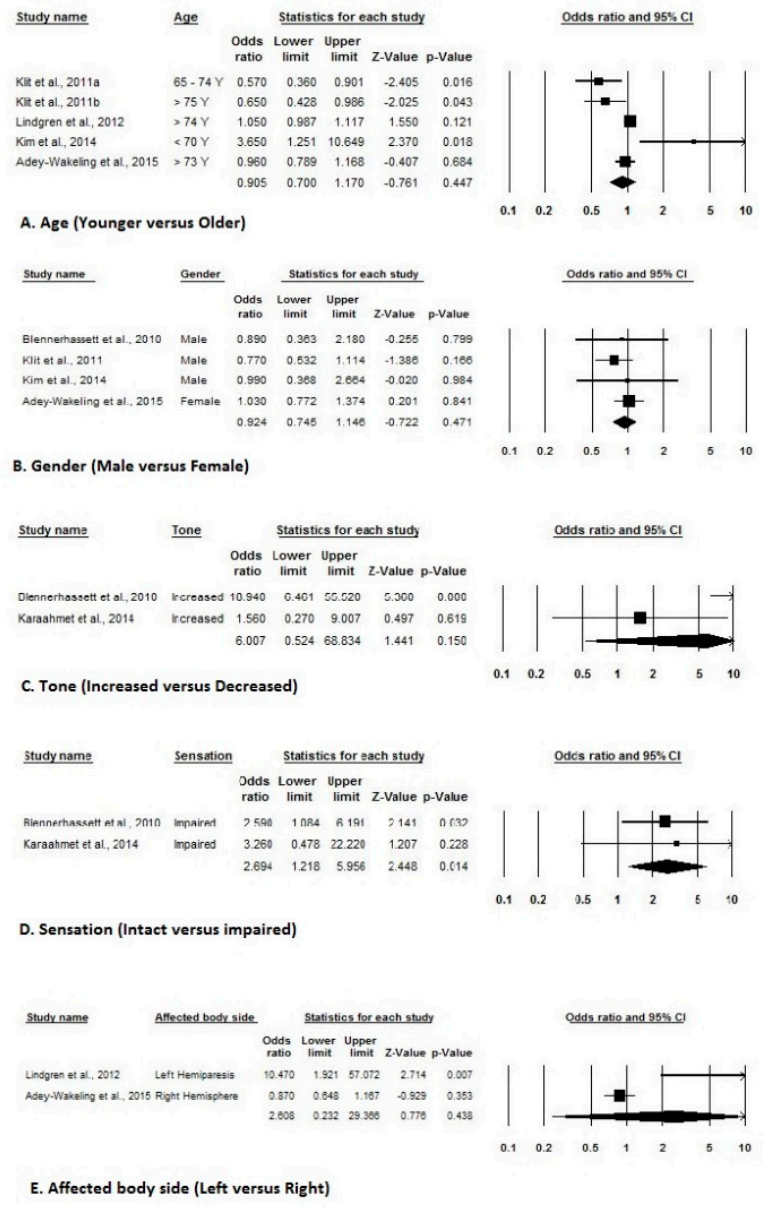

Figure 4. Demographic risk factors for shoulder pain after stroke ((A). Age, (B). Gender, (C). Table 5. Clinical risk factors for shoulder pain after stroke ((A). Types of stroke, (B). Hemispatial neglect, (C). Past medical history, (D). National Institutes of Health Stroke Scale-NIHSS Score). 


\begin{tabular}{|c|c|c|c|c|c|c|}
\hline \multirow[t]{2}{*}{ Study name } & \multirow[t]{2}{*}{ Types of Stroke } & \multicolumn{5}{|c|}{ Statistics for each study } \\
\hline & & $\begin{array}{l}\text { Odds } \\
\text { ratio }\end{array}$ & $\begin{array}{c}\text { Lower } \\
\text { limit }\end{array}$ & $\begin{array}{c}\text { Upper } \\
\text { limit }\end{array}$ & Z-Value & p-Value \\
\hline Blennerhassett et al., 2010 & Infarction & 0.760 & 0.263 & 2.196 & -0.507 & 0.612 \\
\hline Klit et al., 2011 & Infacrtion & 0.730 & 0.428 & 1.250 & -1.147 & 0.251 \\
\hline Adey-Wakeling et sl., 2015 & Hemorrhage & 1.220 & 0.492 & 3.024 & 0.429 & 0.688 \\
\hline & & 0.822 & 0.538 & 1.256 & -0.907 & 0.36 \\
\hline
\end{tabular}

\section{A. Types of stroke (Infarction versus Hemorrhage)}

\section{B. Hemispatial neglect (Yes versus No)}

Odds ratio and $95 \% \mathrm{Cl}$

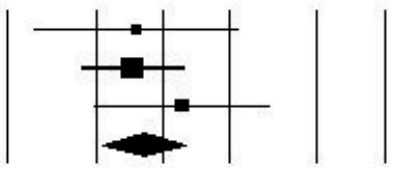

$\begin{array}{lllllll}0.1 & 0.2 & 0.5 & 1 & 2 & 5 & 10\end{array}$

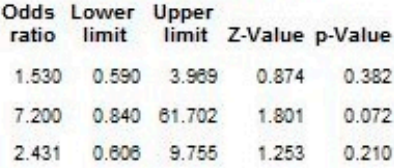

\begin{tabular}{|c|c|}
\hline Study name & Past medical history \\
\hline KII et al, 2011 & Dlaberes \\
\hline Blennernasset et al. 2010 & Prevlous Snoulder Problem \\
\hline Adefe-Wakelling et al., $2015 \mathrm{a}$ & Prevlous Stroke \\
\hline Adley-Wakelling et al., 20150 & Previous Myocarolal intaretion \\
\hline Adej-Wakelling et al, $2015 \mathrm{C}$ & Hypertension \\
\hline Adej-Wakelling et al, 20150 & Dlabenes \\
\hline Adej-Wakelling et al, $2015 e$ & Prevlous Snoulder Pain \\
\hline
\end{tabular}

\begin{tabular}{|c|c|c|c|c|}
\hline \multirow[b]{2}{*}{$\begin{array}{l}\text { Odds } \\
\text { ratio }\end{array}$} & \multicolumn{4}{|c|}{ S.tatistics for each study } \\
\hline & $\begin{array}{l}\text { Lower } \\
\text { limit }\end{array}$ & $\begin{array}{l}\text { Upper } \\
\text { limit }\end{array}$ & Z-Value & te \\
\hline 1.090 & 0.653 & 1.787 & 0299 & \\
\hline 2550 & 33 & 10.271 & & \\
\hline 0.470 & 0.208 & 1.051 & -1.818 & \\
\hline 1.160 & 0.530 & 2539 & 0.371 & \\
\hline 0.960 & 0.499 & 1.847 & -0.122 & \\
\hline 1200 & 0.623 & 2.311 & 0.545 & \\
\hline 8.090 & 3.157 & 20.731 & 4.355 & \\
\hline & 0.776 & 2366 & 1.069 & \\
\hline
\end{tabular}

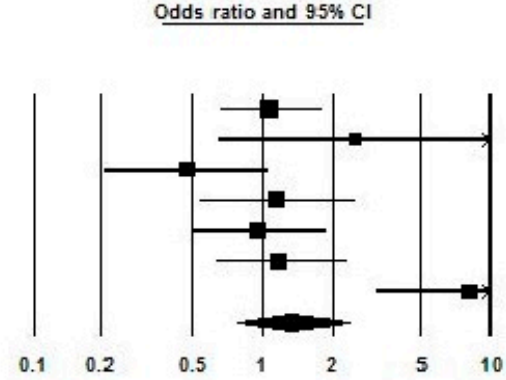

\section{Past Medical History (Yes versus No)}

\begin{tabular}{|c|c|c|c|c|c|c|}
\hline \multirow[t]{2}{*}{ Study name } & \multirow[t]{2}{*}{ NIHSS score } & \multirow[b]{2}{*}{$\begin{array}{r}\text { Odds } \\
\text { ratio }\end{array}$} & \multicolumn{3}{|c|}{ Statistics for each study } & \multirow[b]{2}{*}{ p-Value } \\
\hline & & & $\begin{array}{l}\text { Lower } \\
\text { limit }\end{array}$ & $\begin{array}{c}\text { Upper } \\
\text { limit }\end{array}$ & Z-Value & \\
\hline Kim et al., $2 \mathrm{C} 14$ & Poor & 2.960 & 1.142 & 7.673 & 2.233 & $0 . c 26$ \\
\hline \multirow[t]{2}{*}{ Adey-V/akelirg et al., 2015} & Poor & 1.390 & C. 778 & 2.484 & 1.112 & 0.286 \\
\hline & & 1.839 & c. 899 & 3.760 & 1.689 & $0 . c s$ \\
\hline
\end{tabular}

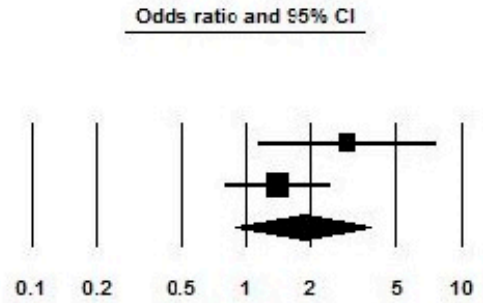

\section{NIHSS Score (Good versus Poor)}

Figure 5. Clinical risk factors for shoulder pain after stroke ((A). Types of stroke, (B). Hemispatial neglect, (C). Past medical history, (D). National Institutes of Health Stroke Scale-NIHSS Score).

\section{Discussion}

Many reviews on HSP have been published in recent decades [41-46]. However, these reviews focus on the cause and treatment of HSP after stroke. As per our knowledge, this is the first systematic review, presenting the results of 18 studies on the incidence and prevalence of HSP after stroke. Additionally, the present review assessed the risk factors causing HSP after stroke. In the current review, a total of 5086 patients with a mean age ranging from 58.7 to 76 years were included. Seven included studies were rated as "good" quality, while one study rated "fair", and 10 studies rated "poor" quality. Eleven studies reported the prevalence, while eight studies reported the incidence rate of HSP following a stroke. The incidence of HSP was ranging from 10 to $22 \%$ in the metanalysis of the included studies. The prevalence of HSP was ranging from 22 to $47 \%$ in the metanalysis of the included studies. 
Previous studies reported the prevalence of shoulder pain after stroke between $21 \%$ and $84 \%$ [ 6 , $12,14,16,30]$, while others reported $5 \%$ and $84 \%[47,48]$. The wide variation in the prevalence of HSP reflects the lack of proper definition or an inconsistency in the quality of care of these patients among diverse populations $[49,50]$. Incidence at admission was approximately $10 \%$ in the present review. The incidence of HSP after a stroke at 6 months was $22 \%$ in the present review. Wanklyn et al. [51] reported that about $63 \%$ of the patients developed HSP in the first six months after stroke. Other studies reported that the HSP usually develops within 2-3 months following the stroke onset $[2,6,16]$. In the current review, 12 months prevalence of HSP after a stroke was $39 \%$. A previous study reported the prevalence of HSP about 32\% within the 12 months after a stroke [52]. Another study reported the prevalence of HSP about $34 \%$ at 12 months after a stroke [36].

In the current review, the most significant identified predictors of HSP were age (younger than 70 years), female gender, increased tone, sensory impairment, left-sided hemiparesis, hemorrhagic stroke, hemispatial neglect, positive past medical history, and poor National Institutes of Health Stroke Scale score. In the previous study, age was not directly linked to the development of HSP, but older people could have preexisting problems that affect shoulder pain [53]. Another study reported various risk factors, including impaired motor control, reduced proprioception, sensory impairment, spasticity of the elbow flexor muscles, limited range of motion (ROM) of the shoulder joint, and type 2 diabetes mellitus [54]. Barlak et al. [13] reported a significant correlation between HSP and complex regional pain syndrome and adhesive capsulitis, but there was no correlation found between the HSP and the grade of subluxation, impingement syndrome or spasticity. Similarly, other studies also reported many risk factors for shoulder pain, and the severity of motor impairment is one of the most important risk factors identified $[6,15,16,40,51,54]$.

During the recovery from stroke, muscle spasticity of the upper extremities is thought to cause shoulder subluxation and limited ROM, resulting in the development of shoulder pain [55]. Another important cause of HSP is frozen shoulder (Adhesive capsulitis), which is indicated by a limited shoulder ROM, with a capsular type of restriction [56-58]. However, other factors could also cause a reduced shoulder ROM in stroke patients without frozen shoulder. Mao et al. [59] identified a prolonged, shortened position was one of the causes of developing soft tissues contracture around the shoulder such as muscles, tendons, and ligaments. The current review was not able to identify a single pathology causing shoulder pain in patients with stroke. This area of research should be explored in the future investigations.

The major strength of this review was the inclusion of a metanalysis, which identified many risk factors causing shoulder pain after stroke. Additionally, most of the included studies were categorized as a prospective observational study, which further enhances the validity of the results. The present study had some potential limitations as well. Some of the included studies did not use a valid and reliable outcome measure to assess symptoms of shoulder pain, which could affect the validity of the results. Additionally, most of the included studies did not report the exact cause of shoulder pain following stroke. Furthermore, variations in the participants' characteristics in the included studies prevent to make any causal relationships between shoulder pain and predictor measures.

\section{Conclusions}

The incidence and prevalence of shoulder pain after stroke vary considerably due to various factors. Knowledge of predictors is important to minimize the risk of developing shoulder pain following a stroke.

Author Contributions: S.A.: the corresponding author; participated in the study design and concept; wrote the review protocol; contributed to the article review, selection and data extraction; contributed to the analysis, interpretation, and presentation of data; and drafted the manuscript. A.A.: participated in the study design and concept; participated in data extraction; contributed to the analysis and provided a critical revision of the manuscript. Both authors approved the final version of the manuscript. All authors have read and agreed to the published version of the manuscript. 
Funding: This research was funded by Deanship of Scientific Research at King Saud University (grant NO. RGP-VPP-209).

Acknowledgments: The authors would like to extend their sincere appreciation to the Deanship of Scientific Research at King Saud University for funding this research through the research group NO. RGP-VPP-209.

Conflicts of Interest: The authors report no conflict of interest.

\section{References}

1. Pan, R.; Zhou, M.; Cai, H.; Guo, Y.; Zhan, L.; Li, M.; Yang, Z.; Zhu, L.; Zhan, J.; Chen, H. A randomized controlled trial of a modified wheelchair arm-support to reduce shoulder pain in stroke patients. Clin. Rehabil. 2017. [CrossRef] [PubMed]

2. Poduri, K.R. Shoulder pain in stroke patients and its effects on rehabilitation. J. Stroke Cerebrovasc. Dis. 1993, 3, 261-266. [CrossRef]

3. Adey-Wakeling, Z.; Arima, H.; Crotty, M.; Leyden, J.; Kleinig, T.; Anderson, C.S.; Newbury, J. Incidence and associations of hemiplegic shoulder pain poststroke: Prospective population-based study. Arch. Phys. Med. Rehabil. 2015, 96, 241-247. [CrossRef] [PubMed]

4. Vasudevan, J.M.; Vasudevan, S.V. Hemiplegic shoulder pain: Diagnosis and management. Crit. Rev. Phys. Rehabil. Med. 2008, 20, 207-220. [CrossRef]

5. Murie-Fernández, M.; Carmona Iragui, M.; Gnanakumar, V.; Meyer, M.; Foley, N.; Teasell, R. Painful hemiplegic shoulder in stroke patients: Causes and management. Neurologia 2012, 27, 234-244. [CrossRef]

6. Lindgren, I.; Jonsson, A.C.; Norrving, B.; Lindgren, A. Shoulder pain after stroke: A prospective population-based study. Stroke 2007, 38, 343-348. [CrossRef]

7. Koog, Y.H.; Jin, S.S.; Yoon, K.; Min, B.I. Interventions for hemiplegic shoulder pain: Systematic review of randomized controlled trials. Disabil. Rehabil. 2010, 32, 282-291. [CrossRef]

8. Bender, L.; McKenna, K. Hemiplegic shoulder pain: Defining the problem and its management. Disabil. Rehabil. 2001, 23, 698-705.

9. Aras, M.; Gokkaya, N.K.; Comert, D.; Kaya, A.; Cakci, A. Shoulder pain in hemiplegia - results from a national rehabilitation hospital in Turkey. Am. J. Phys. Med. Rehabil. 2004, 83, 713-719. [CrossRef] [PubMed]

10. McLean, D.E. Medical complications experienced by a cohort of stroke survivors during inpatient, tertiary-level stroke rehabilitation. Arch. Phys. Med. Rehabil. 2004, 85, 466-469. [CrossRef]

11. Blennerhassett, J.M.; Gyngell, K.; Crean, R. Reduced active control and passive range at the shoulder increase risk of shoulder pain during inpatient rehabilitation post-stroke: An observational study. J. Physiother. 2010, 56, 195-199. [CrossRef]

12. Dromerick, A.W.; Edwards, D.F.; Kumar, A. Hemiplegic shoulder pain syndrome: Frequency and characteristics during inpatient stroke rehabilitation. Arch. Phys. Med. Rehabil. 2008, 89, 1589-1593. [CrossRef] [PubMed]

13. Barlak, A.; Unsal, S.; Kaya, K.; Sahin-Onat, S.; Ozel, S. Poststroke shoulder pain in Turkish stroke patients: Relationship with clinical factors and functional outcomes. Int. J. Rehabil. Res. 2009, 32, 309-315. [CrossRef] [PubMed]

14. Langhorne, P.; Stott, D.J.; Robertson, L.; Macdonald, J.; Jones, L.; McAlpine, C.; Dick, F.; Taylor, G.S.; Murray, G. Medical complications after stroke: A multicenter study. Stroke 2000, 31, 1223-1229. [CrossRef]

15. Ratnasabapathy, Y.; Broad, J.; Baskett, J.; Pledger, M.; Marshall, J.; Bonita, R. Shoulder pain in people with a stroke: A population-based study. Clin. Rehabil. 2003, 17, 304-311. [CrossRef]

16. Gamble, G.E.; Barberan, E.; Laasch, H.U.; Bowsher, D.; Tyrrell, P.J.; Jones, A.K. Post stroke shoulder pain: A prospective study of the association and risk factors in 152 patients from a consecutive cohort of 205 patients presenting with stroke. Eur. J. Pain 2002, 6, 467-474. [CrossRef]

17. Demirci, A.; Öcek, B.; Köseoglu, F. Shoulder pain in hemiplegic patients. J. Phys. Med. Rehabil. Sci. 2007, 1, 25-30.

18. Ikai, T.; Tei, K.; Yoshida, K.; Miyano, S.; Yonemoto, K. Evaluation and treatment of shoulder subluxation in hemiplegia: Relationship between subluxation and pain. Am. J. Phys. Med. Rehabil. 1998, 77, 421-426. [CrossRef]

19. Hanger, H.C.; Whitewood, P.; Brown, G.; Ball, M.C.; Harper, J.; Cox, R.; Sainsbury, R. A randomized controlled trial of strapping to prevent post-stroke shoulder pain. Clin. Rehabil. 2000, 14, 370-380. [CrossRef] 
20. Gustafsson, L.; McKenna, K. A programme of static positional stretches does not reduce hemiplegic shoulder pain or maintain shoulder range of motion-A randomized controlled trial. Clin. Rehabil. 2006, 20, 277-286. [CrossRef]

21. Andrews, A.W.; Bohannon, R.W. Decreased shoulder range of motion on paretic side after stroke. Phys. Ther. 1989, 69, 768-772. [CrossRef] [PubMed]

22. Yamaguchi, K.; Sher, J.S.; Andersen, W.K.; Garretson, R.; Uribe, J.W.; Hechtman, K.; Neviaser, R.J. Glenohumeral motion in patients with rotator cuff tears: A comparison of asymptomatic and symptomatic shoulders. J. Shoulder Elbow Surg. 2000, 9, 6-11. [CrossRef]

23. Chantraine, A.; Baribeault, A.; Uebelhart, D.; Gremion, G. Shoulder pain and dysfunction in hemiplegia: Effects of functional electrical stimulation. Arch. Phys. Med. Rehabil. 1999, 80, 328-331. [CrossRef]

24. Vuagnat, H.; Chantraine, A. Shoulder pain in hemiplegia revisited: Contribution of functional electrical stimulation and other therapies. J. Rehabil. Med. 2003, 35, 49-54. [CrossRef] [PubMed]

25. Meskers, C.G.; Koppe, P.A.; Konijnenbelt, M.H.; Veeger, D.H.; Janssen, T.W. Kinematic alterations in the ipsilateral shoulder of patients with hemiplegia due to stroke. Am. J. Phys. Med. Rehabil. 2005, 84, 97-105. [CrossRef]

26. Stroup, D.F.; Berlin, J.A.; Morton, S.C.; Olkin, I.; Williamson, G.D.; Rennie, D.; Moher, D.; Becker, B.J.; Sipe, T.A.; Thacker, S.B. Meta-analysis of observational studies in epidemiology: A proposal for reporting. JAMA 2000, 283, 2008-2012. [CrossRef]

27. Wells, G.A.; Shea, B.; O'Connell, D.; Peterson, J.; Welch, V.; Losos, M.; Tugwell, P. The Newcastle-Ottawa Scale (NOS) for Assessing the Quality of Nonrandomized Studies in Meta-Analyses; Ottawa Health Research Institute: Ottawa, ON, Canada, 2010.

28. Stang, A. Critical evaluation of the Newcastle-Ottawa scale for the assessment of the quality of nonrandomized studies in meta-analyses. Eur. J. Epidemiol. 2010, 25, 603-605. [CrossRef]

29. Gamble, G.E.; Barberan, E.; Bowsher, D.; Tyrrell, P.J.; Jones, A.K. Post stroke shoulder pain: More common than previously realized. Eur. J. Pain 2000, 4, 313-315. [CrossRef]

30. Sackley, C.; Brittle, N.; Patel, S.; Ellins, J.; Scott, M.; Wright, C.; Dewey, M. The prevalence of joint contractures, pressure sores, painful shoulder, other pain, falls, and depression in the year after a severely disabling stroke. Stroke 2008, 39, 3329-3334. [CrossRef]

31. Kuptniratsaikul, V.; Kovindha, A.; Suethanapornkul, S.; Manimmanakorn, N.; Archongka, Y. Complications during the rehabilitation period in Thai patients with stroke: A multicenter prospective study. Am. J. Phys. Med. Rehabil. 2009, 88, 92-99. [CrossRef]

32. Klit, H.; Finnerup, N.B.; Overvad, K.; Andersen, G.; Jensen, T.S. Pain following stroke: A population-based follow-up study. PLoS ONE 2011, 6, e27607. [CrossRef]

33. Hansen, A.P.; Marcussen, N.S.; Klit, H.; Andersen, G.; Finnerup, N.B.; Jensen, T.S. Pain following stroke: A prospective study. Eur. J. Pain 2012, 16, 1128-1136. [CrossRef] [PubMed]

34. Lindgren, I.; Lexell, J.; Jönsson, A.C.; Brogårdh, C. Left-sided hemiparesis, pain frequency, and decreased passive shoulder range of abduction are predictors of long-lasting poststroke shoulder pain. PMR 2012, 4, 561-568. [CrossRef] [PubMed]

35. Kuptniratsaikul, V.; Kovindha, A.; Suethanapornkul, S.; Manimmanakorn, N.; Archongka, Y. Long-term morbidities in stroke survivors: A prospective multicenter study of Thai stroke rehabilitation registry. BMC Geriatr. 2013, 13, 33. [CrossRef] [PubMed]

36. Chen, C.-M.; Hsu, H.; Chang, C.-H.; Lin, C.-H.; Chen, K.-H.; Hsieh, W.-C.; Chang, W.-M. Age-based prediction of incidence of complications during inpatient stroke rehabilitation: A retrospective longitudinal cohort study. BMC Geriatr. 2014, 14, 41. [CrossRef] [PubMed]

37. Kim, Y.H.; Jung, S.J.; Yang, E.J.; Paik, N.J. Clinical and sonographic risk factors for hemiplegic shoulder pain: A longitudinal observational study. J. Rehabil. Med. 2014, 46, 81-87. [CrossRef]

38. Kwon, Y.H.; Kwon, J.W.; Lee, N.K.; Kang, K.W.; Son, S.M. Prevalence and determinants of pain in the ipsilateral upper limb of stroke patients. Percept. Mot. Skills 2014, 119, 799-810. [CrossRef]

39. Karaahmet, O.Z.; Eksioglu, E.; Gürçay, E.; Karsli, P.B.; Tamkan, U.; Bal, A.; Cakcı, A.; Cakci, A. Hemiplegic shoulder pain: Associated factors and rehabilitation outcomes of hemiplegic patients with and without shoulder pain. Top. Stroke Rehabil. 2014, 21, 237-245. [CrossRef] 
40. Paolucci, S.; Iosa, M.; Barbanti, P.; Bovi, P.; Candeloro, E.; Mancini, A.; Monaco, S.; Pieroni, A.; Truini, A.; Toni, D.; et al. Prevalence and Time Course of Post-Stroke Pain: A Multicenter Prospective Hospital-Based Study. Pain Med. 2016, 17, 924-930. [CrossRef]

41. Griffin, J.W. Hemiplegic shoulder pain. Phys. Ther. 1986, 66, 1884-1893. [CrossRef]

42. Roy, C.W. Shoulder pain in hemiplegia: A literature review. Clin. Rehabil. 1988, 2, 35-44. [CrossRef]

43. Bruton, J.D. Shoulder pain in stroke patients with hemiplegia or hemiparesis following a cerebrovascular accident. Physiotherapy 1985, 71, 2-4.

44. Krotenberg, R. Shoulder pain in hemiplegia. Adv. Clin. Rehabil. 1990, 3, 189-196. [PubMed]

45. Wanklyn, P. The painful hemiplegic shoulder: Pathogenesis, diagnosis and management. Rev. Clin. Gerontol. 1994, 4, 245-251. [CrossRef]

46. Griffin, J.; Reddin, G. Shoulder pain in patients with hemiplegia. A literature review. Phys. Ther. 1981, 61, 1041-1045. [CrossRef]

47. Bohannon, R.W.; Larkin, A.P.; Smith, M.B.; Horton, M.G. Shoulder pain in hemiplegia: Statistical relationship with five variables. Arch. Phys. Med. Rehabil. 1986, 67, 514-516.

48. Coskun Benlidayi, I.; Basaran, S. Hemiplegic shoulder pain: A common clinical consequence of stroke. Pract. Neurol. 2014, 14, 88-91. [CrossRef]

49. Kalichman, L.; Ratmansky, M. Underlying pathology and associated factors of hemiplegic shoulder pain. Am. J. Phys. Med. Rehabil. 2011, 90, 768-780. [CrossRef]

50. Turner-Stokes, L.; Jackson, D. Shoulder pain after stroke: A review of the evidence base to inform the development of an integrated care pathway. Clin. Rehabil. 2002, 16, 276-298. [CrossRef]

51. Wanklyn, P.; Forster, A.; Young, J. Hemiplegic shoulder pain (HSP): Natural history and investigation of associated features. Disabil. Rehabil. 1996, 18, 497-501. [CrossRef]

52. Hadianfard, H.; Hadianfard, M.J. Predictor factors of hemiplegic shoulder pain in a group of stroke patients. Iran Red Crescent Med. J. 2008, 10, 215-219.

53. Vasudevan, J.M.; Browne, B.J. Hemiplegic shoulder pain: An approach to diagnosis and management. Phys. Med. Rehabil. Clin. N. Am. 2014, 25, 411-437. [CrossRef] [PubMed]

54. Roosink, M.; Renzenbrink, G.J.; Buitenweg, J.R.; Van Dongen, R.T.; Geurts, A.C.; IJzerman, M.J. Persistent shoulder pain in the first 6 months after stroke: Results of a prospective cohort study. Arch. Phys. Med. Rehabil. 2011, 92, 1139-1145. [CrossRef] [PubMed]

55. Van Ouwenaller, C.; Laplace, P.M.; Chantraine, A. Painful shoulder in hemiplegia. Arch. Phys. Med. Rehabil. 1986, 67, 23-25.

56. Gillen, G. Upper Extremity Function and Management In Stroke Rehabilitation: A Function-Based Approach; Gillen, G., Burkhardt, A., Eds.; Mosby: St. Louis, MO, USA, 1998; pp. 109-151.

57. O'Sullivan, S.B.; Schmitz, T.J. Physical Rehabilitation: Assessment and Treatment, 3rd ed.; FA Davis: Philadelphia, PA, USA, 1995; pp. 327-360.

58. Iannotti, J.P.; Williams, G.R. Disorders of the Shoulder: Diagnosis and Management; Lippincott Williams \& Wilkins: Philadelphia, PA, USA, 1999.

59. Mao, C.Y.; Jaw, W.C.; Cheng, H.C. Frozen shoulder: Correlation between the response to physical therapy and follow-up shoulder arthrography. Arch. Phys. Med. Rehabil. 1997, 78, 857-859. [CrossRef]

(C) 2020 by the authors. Licensee MDPI, Basel, Switzerland. This article is an open access article distributed under the terms and conditions of the Creative Commons Attribution (CC BY) license (http://creativecommons.org/licenses/by/4.0/). 